Acta Crystallographica Section E

Structure Reports

Online

ISSN 1600-5368

\section{Bis(3-aminopyrazine-2-carboxylato- $\left.\kappa^{2} N^{1}, O\right)$ diaquamanganese(II) mono- hydrate}

\section{Shan $\mathrm{Gao}^{\mathrm{a}}$ and Seik Weng $\mathrm{Ng}^{\mathrm{b}}$ *}

${ }^{a}$ College of Chemistry and Materials Science, Heilongjiang University, Harbin 150080, People's Republic of China, and 'bepartment of Chemistry, University of Malaya, 50603 Kuala Lumpur, Malaysia

Correspondence e-mail: seikweng@um.edu.my

Received 30 August 2010; accepted 1 September 2010

Key indicators: single-crystal X-ray study; $T=293 \mathrm{~K}$; mean $\sigma(\mathrm{C}-\mathrm{C})=0.008 \AA$; $R$ factor $=0.047 ; w R$ factor $=0.165 ;$ data-to-parameter ratio $=13.4$.

In the title compound, $\left[\mathrm{Mn}\left(\mathrm{C}_{5} \mathrm{H}_{4} \mathrm{~N}_{3} \mathrm{O}_{2}\right)_{2}\left(\mathrm{H}_{2} \mathrm{O}\right)_{2}\right] \cdot \mathrm{H}_{2} \mathrm{O}$, the $\mathrm{Mn}^{\mathrm{II}}$ cation, located on a twofold rotation axis, is $\mathrm{N}, \mathrm{O}$-chelated by two 3-aminopyrazine-2-carboxylate anions and coordinated by two water molecules in a distorted octahedral geometry. The uncoordinated water molecules lies on a twofold rotation axis. Adjacent molecules are linked by $\mathrm{O}-$ $\mathrm{H} \cdots \mathrm{O}$ and $\mathrm{N}-\mathrm{H} \cdots \mathrm{O}$ hydrogen bonds into a three-dimensional network motif.

\section{Related literature}

For the isostructural magnesium analog, see: Ptasiewicz-Bak \& Leciejewicz (1997); Marsh (2004).

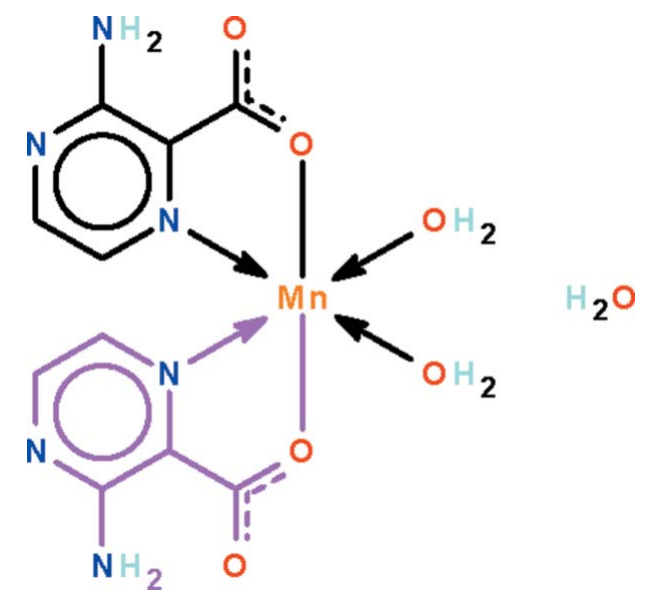

\section{Experimental}

Crystal data

$\left[\mathrm{Mn}\left(\mathrm{C}_{5} \mathrm{H}_{4} \mathrm{~N}_{3} \mathrm{O}_{2}\right)_{2}\left(\mathrm{H}_{2} \mathrm{O}\right)_{2}\right] \cdot \mathrm{H}_{2} \mathrm{O} \quad M_{r}=385.21$
Orthorhombic, Fdd2
$a=8.3107(6) \AA$
$b=29.5862(17) \AA$
$c=12.3791(7) \AA$
$V=3043.8(3) \AA^{3}$

Data collection

Rigaku R-AXIS RAPID diffractometer

Absorption correction: multi-scan (ABSCOR; Higashi, 1995)

$T_{\min }=0.875, T_{\max }=0.930$

Refinement

$R\left[F^{2}>2 \sigma\left(F^{2}\right)\right]=0.047$

$w R\left(F^{2}\right)=0.165$

$S=1.14$

1684 reflections

6 restraints
126 parameters
$Z=8$

Mo $K \alpha$ radiation

$T=293 \mathrm{~K}$

$0.15 \times 0.10 \times 0.08 \mathrm{~mm}$

7239 measured reflections 1684 independent reflections 1086 reflections with $I>2 \sigma(I)$ $R_{\text {int }}=0.056$ $\mu=0.92 \mathrm{~mm}^{-1}$
$\mathrm{H}$ atoms treated by a mixture of independent and constrained refinement
$\Delta \rho_{\max }=0.50{\mathrm{e} \AA^{-3}}^{-3}$

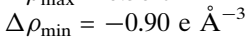
Absolute structure: Flack (1983), 775 Friedel pairs
Flack parameter: -0.02 (5)

Table 1

Hydrogen-bond geometry $\left(\AA{ }^{\circ}\right)$.

\begin{tabular}{lllll}
\hline$D-\mathrm{H} \cdots A$ & $D-\mathrm{H}$ & $\mathrm{H} \cdots A$ & $D \cdots A$ & $D-\mathrm{H} \cdots A$ \\
\hline $\mathrm{O} 1 \mathrm{w}-\mathrm{H} 11 \cdots \mathrm{O} 2{ }^{\mathrm{i}}$ & $0.84(7)$ & $1.89(3)$ & $2.704(7)$ & $162(9)$ \\
$\mathrm{O} 1 \mathrm{w}-\mathrm{H} 12 \cdots \mathrm{N} 2^{\mathrm{ii}}$ & $0.84(7)$ & $2.02(4)$ & $2.792(7)$ & $152(9)$ \\
$\mathrm{O} 2 \mathrm{w}-\mathrm{H} 2 \cdots \mathrm{O} 1$ & $0.84(7)$ & $2.10(4)$ & $2.902(7)$ & $159(10)$ \\
$\mathrm{N} 3-\mathrm{H} 31 \cdots \mathrm{O} 2$ & $0.88(7)$ & $2.17(9)$ & $2.690(8)$ & $118(8)$ \\
$\mathrm{N} 3-\mathrm{H} 32 \cdots \mathrm{O} 2 \mathrm{w}^{\text {iii }}$ & $0.88(3)$ & $2.15(3)$ & $3.001(7)$ & $161(9)$ \\
\hline \multicolumn{2}{l}{ Symmetry codes: (i) $-x,-y+\frac{1}{2}, z+\frac{1}{2} ;$ (ii) $x-\frac{1}{4},-y+\frac{3}{4}, z+\frac{1}{4} ;$ (iii) $-x-\frac{1}{4}, y+\frac{1}{4}, z-\frac{1}{4}}$.
\end{tabular}

Data collection: RAPID-AUTO (Rigaku, 1998); cell refinement: RAPID-AUTO; data reduction: CrystalStructure (Rigaku/MSC, 2002); program(s) used to solve structure: SHELXS97 (Sheldrick, 2008); $\operatorname{program}(\mathrm{s})$ used to refine structure: SHELXL97 (Sheldrick, 2008); molecular graphics: $X$-SEED (Barbour, 2001); software used to prepare material for publication: publCIF (Westrip, 2010).

We thank the Key Project of the Natural Science Foundation of Heilongjiang Province (No. ZD200903), the Innovation Team of the Education Bureau of Heilongjiang Province (No. $2010 \mathrm{t}$ d03), Heilongjiang University and the University of Malaya for supporting this study.

Supplementary data and figures for this paper are available from the IUCr electronic archives (Reference: XU5022).

\section{References}

Barbour, L. J. (2001). J. Supramol. Chem. 1, 189-191.

Flack, H. D. (1983). Acta Cryst. A39, 876-881.

Higashi, T. (1995). ABSCOR. Rigaku Corporation, Tokyo, Japan.

Marsh, R. E. (2004). Acta Cryst. B60, 252-253.

Ptasiewicz-Bak, H. \& Leciejewicz, J. (1997). Pol. J. Chem. 71, 1350-1358.

Rigaku (1998). RAPID-AUTO. Rigaku Corporation, Tokyo, Japan.

Rigaku/MSC (2002). CrystalStructure. Rigaku/MSC, The Woodlands, Texas, USA.

Sheldrick, G. M. (2008). Acta Cryst. A64, 112-122.

Westrip, S. P. (2010). J. Appl. Cryst. 43, 920-925. 


\section{supporting information}

Acta Cryst. (2010). E66, m1223 [doi:10.1107/S1600536810035233]

\section{Bis(3-aminopyrazine-2-carboxylato- $\kappa^{2} N^{1}, O$ ) diaquamanganese(II) monohydrate}

\section{Shan Gao and Seik Weng Ng}

\section{S1. Comment}

The crystal structure of $\mathrm{Mg}\left(\mathrm{H}_{2} \mathrm{O}\right)_{2}\left(\mathrm{C}_{5} \mathrm{H}_{4} \mathrm{~N}_{3} \mathrm{O}_{2}\right)_{2} \mathrm{H}_{2} \mathrm{O}$ was described in the $C c$ space group (Ptasiewicz-Bak \& Leciejewicz, 1997); the space group was revised to the $F d d 2$ space group (Marsh, 2004). The manganese analog (Scheme I) is isostructural; The water-coordinated manganese atom is $\mathrm{N}, \mathrm{O}$-chelated by the carboxylate ion (Fig. 2) in an octahedral environment. The mononuclear and lattice water both lie on a twofold rotation axis. Adjacent molecules are linked by $\mathrm{O}_{-}$ $\mathrm{H} \cdots \mathrm{O}$ and $\mathrm{N}-\mathrm{H} \cdots \mathrm{O}$ hydrogen bonds into a three-dimensional network motif.

\section{S2. Experimental}

Manganese acetate $(1 \mathrm{mmol})$ and 2-aminopyrazine-3-carboxylic acid $(2 \mathrm{mmol})$ and sodium hydroxide $(2 \mathrm{mmol})$ were dissolved in a small volume of water to give a light yellow solution. Prismatic crystals separated from the solution after a few days.

\section{S3. Refinement}

Carbon-bound H-atoms were placed in calculated positions ( $\mathrm{C}-\mathrm{H} 0.93 \AA)$ and were included in the refinement in the riding model approximation, with $U(\mathrm{H})$ set to $1.2 U(\mathrm{C})$.

The amino $\mathrm{H}$-atoms and water $\mathrm{H}$-atoms were located in a difference Fourier map, and were refined with a distance restraints of $\mathrm{N}-\mathrm{H} 0.88 \pm 0.01$ and $\mathrm{O}-\mathrm{H} 0.84 \pm 0.01 \AA$; their temperature factors were tied to those of the parent atoms by a factor of 1.5 times.

The final difference Fourier map was featureless.

The second value in the WGHT is somewhat large. Using a smaller value led to a deeper hole in the final difference Fourier map and a larger Goodness-of-fit. 


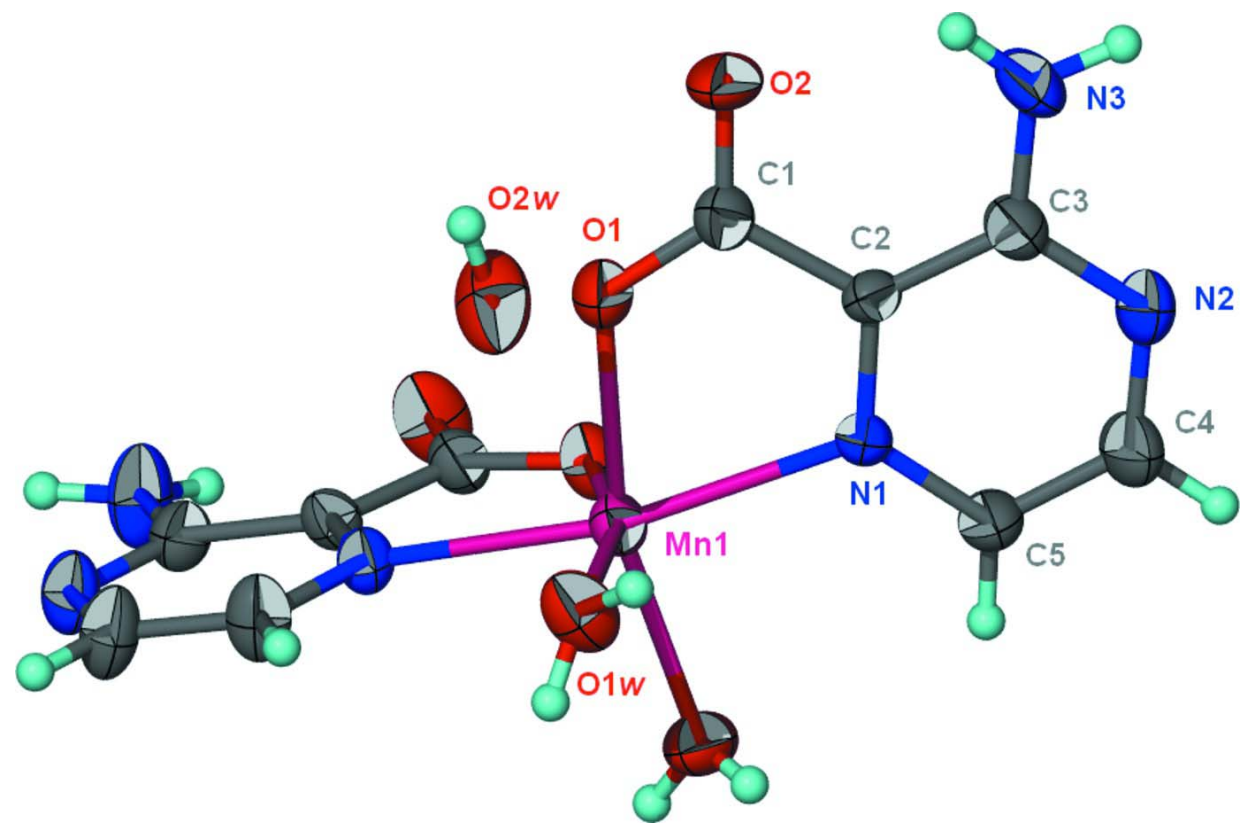

\section{Figure 1}

Thermal ellipsoid plot (Barbour, 2001) of $\mathrm{Mn}\left(\mathrm{H}_{2} \mathrm{O}\right)_{2}\left(\mathrm{C}_{5} \mathrm{H}_{4} \mathrm{~N}_{3} \mathrm{O}_{2}\right)_{2} \mathrm{H}_{2} \mathrm{O}$ at the $50 \%$ probability level; hydrogen atoms are drawn as spheres of arbitrary radius. The $\mathrm{Mn}$ and lattice water molecule lie on a twofold rotation axis. Symmetry-related atoms are not labeled.

\section{Bis(3-aminopyrazine-2-carboxylato- $\left.\kappa^{2} N^{1}, O\right)$ diaquamanganese(II) monohydrate}

\section{Crystal data}

$\left[\mathrm{Mn}\left(\mathrm{C}_{5} \mathrm{H}_{4} \mathrm{~N}_{3} \mathrm{O}_{2}\right)_{2}\left(\mathrm{H}_{2} \mathrm{O}\right)_{2}\right] \cdot \mathrm{H}_{2} \mathrm{O}$

$M_{r}=385.21$

Orthorhombic, $F d d 2$

Hall symbol: F $2-2 \mathrm{~d}$

$a=8.3107(6) \AA$

$b=29.5862(17) \AA$

$c=12.3791(7) \AA$

$V=3043.8(3) \AA^{3}$

$Z=8$

\section{Data collection}

Rigaku R-AXIS RAPID diffractometer

Radiation source: fine-focus sealed tube Graphite monochromator

Detector resolution: 10.000 pixels $\mathrm{mm}^{-1}$

$\omega$ scans

Absorption correction: multi-scan

(ABSCOR; Higashi, 1995)

$T_{\min }=0.875, T_{\max }=0.930$
$F(000)=1576$

$D_{\mathrm{x}}=1.681 \mathrm{Mg} \mathrm{m}^{-3}$

Mo $K \alpha$ radiation, $\lambda=0.71073 \AA$

Cell parameters from 4731 reflections

$\theta=3.0-27.4^{\circ}$

$\mu=0.92 \mathrm{~mm}^{-1}$

$T=293 \mathrm{~K}$

Prism, yellow

$0.15 \times 0.10 \times 0.08 \mathrm{~mm}$

7239 measured reflections

1684 independent reflections

1086 reflections with $I>2 \sigma(I)$

$R_{\text {int }}=0.056$

$\theta_{\max }=27.4^{\circ}, \theta_{\min }=3.0^{\circ}$

$h=-10 \rightarrow 10$

$k=-38 \rightarrow 38$

$l=-16 \rightarrow 15$ 


\section{Refinement}

Refinement on $F^{2}$

Least-squares matrix: full

$R\left[F^{2}>2 \sigma\left(F^{2}\right)\right]=0.047$

$w R\left(F^{2}\right)=0.165$

$S=1.14$

1684 reflections

126 parameters

6 restraints

Primary atom site location: structure-invariant direct methods

Secondary atom site location: difference Fourier map

Hydrogen site location: inferred from neighbouring sites
$\mathrm{H}$ atoms treated by a mixture of independent and constrained refinement

$w=1 /\left[\sigma^{2}\left(F_{\mathrm{o}}^{2}\right)+(0.0722 P)^{2}+15.3101 P\right]$ where $P=\left(F_{\mathrm{o}}^{2}+2 F_{\mathrm{c}}^{2}\right) / 3$

$(\Delta / \sigma)_{\max }=0.001$

$\Delta \rho_{\max }=0.50 \mathrm{e} \AA^{-3}$

$\Delta \rho_{\min }=-0.90$ e $\AA^{-3}$

Extinction correction: SHELXL97 (Sheldrick, 2008), $\mathrm{Fc}^{*}=\mathrm{kFc}\left[1+0.001 \times \mathrm{xc}^{2} \lambda^{3} / \sin (2 \theta)\right]^{-1 / 4}$

Extinction coefficient: 0.0014 (3)

Absolute structure: Flack (1983), 775 Friedel pairs

Absolute structure parameter: -0.02 (5)

Fractional atomic coordinates and isotropic or equivalent isotropic displacement parameters $\left(\hat{A}^{2}\right)$

\begin{tabular}{lllll}
\hline & $x$ & $y$ & $z$ & $U_{\text {iso }}^{*} / U_{\text {eq }}$ \\
\hline Mn1 & 0.2500 & 0.2500 & $0.53687(11)$ & $0.0378(4)$ \\
O1 & $0.0649(6)$ & $0.26569(16)$ & $0.4190(4)$ & $0.0457(11)$ \\
O2 & $-0.0500(7)$ & $0.31796(16)$ & $0.3169(4)$ & $0.0606(16)$ \\
O1W & $0.0675(8)$ & $0.24512(17)$ & $0.6593(4)$ & $0.0561(16)$ \\
H11 & $0.084(11)$ & $0.225(2)$ & $0.706(5)$ & $0.084^{*}$ \\
H12 & $0.006(9)$ & $0.267(2)$ & $0.675(8)$ & $0.084^{*}$ \\
O2W & -0.2500 & 0.2500 & $0.5133(9)$ & $0.069(3)$ \\
H2 & $-0.164(7)$ & $0.248(4)$ & $0.478(7)$ & $0.104^{*}$ \\
N1 & $0.2472(6)$ & $0.32757(14)$ & $0.5175(4)$ & $0.0348(13)$ \\
N2 & $0.2176(7)$ & $0.41941(18)$ & $0.4774(5)$ & $0.0515(16)$ \\
N3 & $0.0251(9)$ & $0.4054(2)$ & $0.3489(6)$ & $0.0634(19)$ \\
H31 & $-0.048(9)$ & $0.391(3)$ & $0.311(7)$ & $0.095^{*}$ \\
H32 & $0.035(11)$ & $0.4344(8)$ & $0.335(8)$ & $0.095^{*}$ \\
C1 & $0.0468(8)$ & $0.30645(19)$ & $0.3886(5)$ & $0.0409(14)$ \\
C2 & $0.1439(7)$ & $0.34192(19)$ & $0.4439(5)$ & $0.0349(12)$ \\
C3 & $0.1271(8)$ & $0.3890(2)$ & $0.4229(5)$ & $0.0427(14)$ \\
C4 & $0.3184(10)$ & $0.4035(2)$ & $0.5501(7)$ & $0.0587(19)$ \\
H4 & 0.3808 & 0.4241 & 0.5886 & $0.070^{*}$ \\
C5 & $0.3368(9)$ & $0.3572(2)$ & $0.5727(6)$ & $0.0503(17)$ \\
H5 & 0.4095 & 0.3474 & 0.6248 & $0.060^{*}$ \\
& & & & \\
\hline
\end{tabular}

Atomic displacement parameters $\left(\AA^{2}\right)$

\begin{tabular}{lllllll}
\hline & $U^{11}$ & $U^{22}$ & $U^{33}$ & $U^{12}$ & $U^{13}$ & $U^{23}$ \\
\hline Mn1 & $0.0457(7)$ & $0.0301(6)$ & $0.0376(7)$ & $0.0012(7)$ & 0.000 & 0.000 \\
O1 & $0.051(3)$ & $0.035(2)$ & $0.051(3)$ & $-0.001(2)$ & $-0.009(2)$ & $0.000(2)$ \\
O2 & $0.076(4)$ & $0.050(2)$ & $0.055(4)$ & $0.010(2)$ & $-0.035(3)$ & $0.000(2)$ \\
O1W & $0.070(4)$ & $0.048(3)$ & $0.050(3)$ & $0.010(3)$ & $0.019(3)$ & $0.008(2)$ \\
O2W & $0.044(4)$ & $0.055(4)$ & $0.109(10)$ & $-0.009(4)$ & 0.000 & 0.000 \\
N1 & $0.041(2)$ & $0.030(2)$ & $0.033(4)$ & $-0.001(2)$ & $-0.009(3)$ & $0.003(2)$ \\
N2 & $0.056(4)$ & $0.039(3)$ & $0.059(4)$ & $-0.015(3)$ & $-0.008(3)$ & $0.004(3)$
\end{tabular}




\begin{tabular}{lllllll}
$\mathrm{N} 3$ & $0.075(5)$ & $0.042(3)$ & $0.073(5)$ & $-0.001(3)$ & $-0.029(4)$ & $0.019(3)$ \\
$\mathrm{C} 1$ & $0.050(4)$ & $0.033(3)$ & $0.040(3)$ & $0.001(3)$ & $-0.001(3)$ & $-0.003(3)$ \\
$\mathrm{C} 2$ & $0.041(3)$ & $0.034(3)$ & $0.030(3)$ & $0.002(2)$ & $-0.008(3)$ & $-0.003(2)$ \\
$\mathrm{C} 3$ & $0.046(4)$ & $0.037(3)$ & $0.044(4)$ & $-0.001(3)$ & $-0.001(3)$ & $0.006(3)$ \\
$\mathrm{C} 4$ & $0.063(4)$ & $0.045(4)$ & $0.068(5)$ & $-0.019(3)$ & $-0.014(4)$ & $0.013(4)$ \\
$\mathrm{C} 5$ & $0.055(4)$ & $0.043(3)$ & $0.053(4)$ & $-0.007(3)$ & $-0.021(3)$ & $0.006(3)$ \\
\hline
\end{tabular}

Geometric parameters $\left(\AA,{ }^{o}\right)$

\begin{tabular}{|c|c|c|c|}
\hline $\mathrm{Mn} 1-\mathrm{O} 1 \mathrm{~W}^{\mathrm{i}}$ & $2.149(6)$ & $\mathrm{N} 1-\mathrm{C} 5$ & $1.338(8)$ \\
\hline $\mathrm{Mn} 1-\mathrm{O} 1 \mathrm{~W}$ & $2.149(6)$ & $\mathrm{N} 2-\mathrm{C} 4$ & $1.316(10)$ \\
\hline $\mathrm{Mn} 1-\mathrm{O} 1$ & $2.170(5)$ & $\mathrm{N} 2-\mathrm{C} 3$ & $1.352(8)$ \\
\hline $\mathrm{Mn} 1-\mathrm{O} 1^{\mathrm{i}}$ & $2.170(5)$ & $\mathrm{N} 3-\mathrm{C} 3$ & $1.339(8)$ \\
\hline $\mathrm{Mn} 1-\mathrm{N} 1^{\mathrm{i}}$ & $2.308(4)$ & N3-H31 & $0.88(7)$ \\
\hline $\mathrm{Mn} 1-\mathrm{N} 1$ & $2.308(4)$ & $\mathrm{N} 3-\mathrm{H} 32$ & $0.88(3)$ \\
\hline $\mathrm{O} 1-\mathrm{C} 1$ & $1.273(7)$ & $\mathrm{C} 1-\mathrm{C} 2$ & $1.490(8)$ \\
\hline $\mathrm{O} 2-\mathrm{C} 1$ & $1.245(8)$ & $\mathrm{C} 2-\mathrm{C} 3$ & $1.424(8)$ \\
\hline $\mathrm{O} 1 \mathrm{~W}-\mathrm{H} 11$ & $0.84(7)$ & $\mathrm{C} 4-\mathrm{C} 5$ & $1.409(9)$ \\
\hline $\mathrm{O} 1 \mathrm{~W}-\mathrm{H} 12$ & $0.84(7)$ & $\mathrm{C} 4-\mathrm{H} 4$ & 0.9300 \\
\hline $\mathrm{O} 2 \mathrm{~W}-\mathrm{H} 2$ & $0.84(7)$ & $\mathrm{C} 5-\mathrm{H} 5$ & 0.9300 \\
\hline $\mathrm{N} 1-\mathrm{C} 2$ & $1.322(7)$ & & \\
\hline $\mathrm{O} 1 \mathrm{~W}-\mathrm{Mn} 1-\mathrm{O} 1 \mathrm{~W}$ & $90.3(4)$ & $\mathrm{C} 5-\mathrm{N} 1-\mathrm{Mn} 1$ & $126.3(4)$ \\
\hline $\mathrm{O} 1 \mathrm{~W}^{\mathrm{i}}-\mathrm{Mn} 1-\mathrm{O} 1$ & $163.73(16)$ & $\mathrm{C} 4-\mathrm{N} 2-\mathrm{C} 3$ & $117.2(6)$ \\
\hline $\mathrm{O} 1 \mathrm{~W}-\mathrm{Mn} 1-\mathrm{O} 1$ & $89.33(18)$ & $\mathrm{C} 3-\mathrm{N} 3-\mathrm{H} 31$ & $129(7)$ \\
\hline $\mathrm{O} 1 \mathrm{~W}^{\mathrm{i}}-\mathrm{Mn} 1-\mathrm{O}^{\mathrm{i}}$ & $89.33(18)$ & $\mathrm{C} 3-\mathrm{N} 3-\mathrm{H} 32$ & $115(6)$ \\
\hline $\mathrm{O} 1 \mathrm{~W}-\mathrm{Mn} 1-\mathrm{O}^{\mathrm{i}}$ & $163.73(16)$ & $\mathrm{H} 31-\mathrm{N} 3-\mathrm{H} 32$ & $115(9)$ \\
\hline $\mathrm{O} 1-\mathrm{Mn} 1-\mathrm{O} 1^{\mathrm{i}}$ & $95.5(3)$ & $\mathrm{O} 2-\mathrm{C} 1-\mathrm{O} 1$ & $123.1(6)$ \\
\hline $\mathrm{O} 1 \mathrm{~W}^{\mathrm{i}}-\mathrm{Mn} 1-\mathrm{N} 1^{\mathrm{i}}$ & $97.65(18)$ & $\mathrm{O} 2-\mathrm{C} 1-\mathrm{C} 2$ & $119.0(5)$ \\
\hline $\mathrm{O} 1 \mathrm{~W}-\mathrm{Mn} 1-\mathrm{N} 1^{\mathrm{i}}$ & $90.79(18)$ & $\mathrm{O} 1-\mathrm{C} 1-\mathrm{C} 2$ & $117.9(6)$ \\
\hline $\mathrm{O} 1-\mathrm{Mn} 1-\mathrm{N} 1^{\mathrm{i}}$ & $98.61(18)$ & $\mathrm{N} 1-\mathrm{C} 2-\mathrm{C} 3$ & $120.2(5)$ \\
\hline $\mathrm{O} 1^{\mathrm{i}}-\mathrm{Mn} 1-\mathrm{N} 1^{\mathrm{i}}$ & $73.16(17)$ & $\mathrm{N} 1-\mathrm{C} 2-\mathrm{C} 1$ & $116.2(5)$ \\
\hline $\mathrm{O} 1 \mathrm{~W}^{\mathrm{i}}-\mathrm{Mn} 1-\mathrm{N} 1$ & $90.79(18)$ & $\mathrm{C} 3-\mathrm{C} 2-\mathrm{C} 1$ & $123.5(5)$ \\
\hline $\mathrm{O} 1 \mathrm{~W}-\mathrm{Mn} 1-\mathrm{N} 1$ & $97.65(18)$ & $\mathrm{N} 3-\mathrm{C} 3-\mathrm{N} 2$ & $116.9(6)$ \\
\hline $\mathrm{O} 1-\mathrm{Mn} 1-\mathrm{N} 1$ & $73.16(17)$ & $\mathrm{N} 3-\mathrm{C} 3-\mathrm{C} 2$ & $122.7(6)$ \\
\hline $\mathrm{O} 1{ }^{\mathrm{i}}-\mathrm{Mn} 1-\mathrm{N} 1$ & $98.61(18)$ & $\mathrm{N} 2-\mathrm{C} 3-\mathrm{C} 2$ & $120.3(6)$ \\
\hline $\mathrm{N} 1{ }^{\mathrm{i}}-\mathrm{Mn} 1-\mathrm{N} 1$ & $168.0(3)$ & $\mathrm{N} 2-\mathrm{C} 4-\mathrm{C} 5$ & $123.5(7)$ \\
\hline $\mathrm{C} 1-\mathrm{O} 1-\mathrm{Mn} 1$ & $119.0(4)$ & $\mathrm{N} 2-\mathrm{C} 4-\mathrm{H} 4$ & 118.2 \\
\hline $\mathrm{Mn} 1-\mathrm{O} 1 \mathrm{~W}-\mathrm{H} 11$ & $115(6)$ & $\mathrm{C} 5-\mathrm{C} 4-\mathrm{H} 4$ & 118.2 \\
\hline $\mathrm{Mn} 1-\mathrm{O} 1 \mathrm{~W}-\mathrm{H} 12$ & $122(7)$ & $\mathrm{N} 1-\mathrm{C} 5-\mathrm{C} 4$ & $118.4(6)$ \\
\hline $\mathrm{H} 11-\mathrm{O} 1 \mathrm{~W}-\mathrm{H} 12$ & $119(10)$ & $\mathrm{N} 1-\mathrm{C} 5-\mathrm{H} 5$ & 120.8 \\
\hline $\mathrm{C} 2-\mathrm{N} 1-\mathrm{C} 5$ & $120.2(5)$ & $\mathrm{C} 4-\mathrm{C} 5-\mathrm{H} 5$ & 120.8 \\
\hline $\mathrm{C} 2-\mathrm{N} 1-\mathrm{Mn} 1$ & $113.4(4)$ & & \\
\hline $\mathrm{O} 1 \mathrm{~W}^{\mathrm{i}}-\mathrm{Mn} 1-\mathrm{O} 1-\mathrm{C} 1$ & $14.2(12)$ & $\mathrm{Mn} 1-\mathrm{N} 1-\mathrm{C} 2-\mathrm{C} 3$ & $179.6(5)$ \\
\hline $\mathrm{O} 1 \mathrm{~W}-\mathrm{Mn} 1-\mathrm{O} 1-\mathrm{C} 1$ & $102.9(5)$ & $\mathrm{C} 5-\mathrm{N} 1-\mathrm{C} 2-\mathrm{C} 1$ & $-178.6(6)$ \\
\hline $\mathrm{O} 1-\mathrm{Mn} 1-\mathrm{O} 1-\mathrm{C} 1$ & $-92.7(5)$ & $\mathrm{Mn} 1-\mathrm{N} 1-\mathrm{C} 2-\mathrm{C} 1$ & $1.0(7)$ \\
\hline $\mathrm{N} 1{ }^{\mathrm{i}}-\mathrm{Mn} 1-\mathrm{O} 1-\mathrm{C} 1$ & $-166.4(5)$ & $\mathrm{O} 2-\mathrm{C} 1-\mathrm{C} 2-\mathrm{N} 1$ & $-178.5(6)$ \\
\hline
\end{tabular}




$\begin{array}{llll}\mathrm{N} 1-\mathrm{Mn} 1-\mathrm{O} 1-\mathrm{C} 1 & 4.7(5) & \mathrm{O} 1-\mathrm{C} 1-\mathrm{C} 2-\mathrm{N} 1 & 3.0(9) \\ \mathrm{O} 1 \mathrm{~W}-\mathrm{Mn} 1-\mathrm{N} 1-\mathrm{C} 2 & 179.9(4) & \mathrm{O} 2-\mathrm{C} 1-\mathrm{C} 2-\mathrm{C} 3 & 3.0(9) \\ \mathrm{O} 1 \mathrm{~W}-\mathrm{Mn} 1-\mathrm{N} 1-\mathrm{C} 2 & -89.8(4) & \mathrm{O} 1-\mathrm{C} 1-\mathrm{C} 2-\mathrm{C} 3 & -175.5(6) \\ \mathrm{O} 1-\mathrm{Mn} 1-\mathrm{N} 1-\mathrm{C} 2 & -2.8(4) & \mathrm{C} 4-\mathrm{N} 2-\mathrm{C} 3-\mathrm{N} 3 & -179.9(8) \\ \mathrm{O} 1-\mathrm{M} 1-\mathrm{N} 1-\mathrm{C} 2 & 90.4(4) & \mathrm{C} 4-\mathrm{N} 2-\mathrm{C} 3-\mathrm{C} 2 & -0.7(10) \\ \mathrm{N} 1-\mathrm{M} 1-\mathrm{N} 1-\mathrm{C} 2 & 44.8(4) & \mathrm{N} 1-\mathrm{C} 2-\mathrm{C} 3-\mathrm{N} 3 & 179.6(7) \\ \mathrm{O} 1 \mathrm{~W}-\mathrm{Mn} 1-\mathrm{N} 1-\mathrm{C} 5 & -0.6(6) & \mathrm{C} 1-\mathrm{C} 2-\mathrm{C} 3-\mathrm{N} 3 & -1.9(10) \\ \mathrm{O} 1 \mathrm{~W}-\mathrm{Mn} 1-\mathrm{N} 1-\mathrm{C} 5 & 89.8(6) & \mathrm{N} 1-\mathrm{C} 2-\mathrm{C} 3-\mathrm{N} 2 & 0.5(10) \\ \mathrm{O} 1-\mathrm{Mn} 1-\mathrm{N} 1-\mathrm{C} 5 & 176.8(6) & \mathrm{C} 1-\mathrm{C} 2-\mathrm{C} 3-\mathrm{N} 2 & 178.9(6) \\ \mathrm{O} 1-\mathrm{Mn} 1-\mathrm{N} 1-\mathrm{C} 5 & -90.0(6) & \mathrm{C} 3-\mathrm{N} 2-\mathrm{C} 4-\mathrm{C} 5 & 0.5(13) \\ \mathrm{N} 1-\mathrm{Mn} 1-\mathrm{N} 1-\mathrm{C} 5 & -135.6(5) & \mathrm{C} 2-\mathrm{N} 1-\mathrm{C} 5-\mathrm{C} 4 & -0.2(10) \\ \mathrm{Mn} 1-\mathrm{O} 1-\mathrm{C} 1-\mathrm{O} 2 & 175.7(5) & \mathrm{Mn} 1-\mathrm{N} 1-\mathrm{C} 5-\mathrm{C} 4 & -179.8(5) \\ \mathrm{Mn} 1-\mathrm{O} 1-\mathrm{C} 1-\mathrm{C} 2 & -5.8(8) & \mathrm{N} 2-\mathrm{C} 4-\mathrm{C} 5-\mathrm{N} 1 & 0.0(13) \\ \mathrm{C} 5-\mathrm{N} 1-\mathrm{C} 2-\mathrm{C} 3 & 0.0(9) & & \end{array}$

Symmetry code: (i) $-x+1 / 2,-y+1 / 2, z$.

Hydrogen-bond geometry $\left(A,{ }^{\circ}\right)$

\begin{tabular}{lllll}
\hline$D-\mathrm{H} \cdots A$ & $D-\mathrm{H}$ & $\mathrm{H} \cdots A$ & $D \cdots A$ & $D-\mathrm{H} \cdots A$ \\
\hline $\mathrm{O} 1 \mathrm{w}-\mathrm{H} 11 \cdots \mathrm{O} 2^{\mathrm{ii}}$ & $0.84(7)$ & $1.89(3)$ & $2.704(7)$ & $162(9)$ \\
$\mathrm{O} 1 \mathrm{w}-\mathrm{H} 12 \cdots \mathrm{N} 2^{\mathrm{iii}}$ & $0.84(7)$ & $2.02(4)$ & $2.792(7)$ & $152(9)$ \\
$\mathrm{O} 2 \mathrm{w}-\mathrm{H} 2 \cdots \mathrm{O} 1$ & $0.84(7)$ & $2.10(4)$ & $2.902(7)$ & $159(10)$ \\
$\mathrm{N} 3-\mathrm{H} 31 \cdots \mathrm{O} 2$ & $0.88(7)$ & $2.17(9)$ & $2.690(8)$ & $118(8)$ \\
$\mathrm{N} 3-\mathrm{H} 32 \cdots \mathrm{O} 2 \mathrm{w}^{\mathrm{iv}}$ & $0.88(3)$ & $2.15(3)$ & $3.001(7)$ & $161(9)$ \\
\hline
\end{tabular}

Symmetry codes: (ii) $-x,-y+1 / 2, z+1 / 2$; (iii) $x-1 / 4,-y+3 / 4, z+1 / 4$; (iv) $-x-1 / 4, y+1 / 4, z-1 / 4$. 